\section{Laser Scanning Cytome- try to Quantify Gene Transfer Efficiency and Transcriptional Activity of EGFP Constructs}

\author{
BioTechniques 32:62-66 (January 2002)
}

\begin{abstract}
Enhanced green fluorescent protein $(E G F P)$ is the preferred reporter protein for real-time detection in individual cells, but its usefulness for gene expression quantification is limited by the sensitivity of standard detection techniques. We tested whether the unique feature of single-cell detection and quantification by laser scanning cytometry permits the evaluation of EGFP gene expression in monolayer cultures of kidney epithelial 293 and C2C12 muscle cells. Cells were transfected with plasmids expressing EGFP under the control of either the cytomegalovirus or muscle promoters: namely, muscle creatine kinase (MCK) and muscle glycogen phosphorylase (MGP). Cell monolayers were laserscanned, fluorescence-imaged, and recorded. A population of fluorescence-emitting cells was discriminated, their contour area was defined, and the integrated fluorescence was estimated. These data were used to assess gene transfer efficiency in cells transfected with CMV-EGFP, which was higher in 293 than in C2C12 cells. Analysis of fluorescence intensity revealed that, as expected, CMV constructs were highly expressed in both cell types, whereas MCK and MGP constructs showed the highest transcriptional activity in C12C12 cells. In summary, we describe the utility of laser scanning cytometry for the automated estimation of gene transfer efficiency and transcriptional activity of EGFP constructs in cell monolayers.
\end{abstract}

\section{INTRODUCTION}

The introduction of GFP as a reporter protein has fostered real-time detection of the delivered gene expression in living cells and tissues by examination under a fluorescence microscope
(2). Moreover, the sensitivity of detection has increased with the development of GFP mutants such as enhanced GFP (EGFP) (7), which bears doubleamino acid substitutions (e.g., Phe-64 to Leu and Ser-65 to Thr) and exhibits 35 -fold higher fluorescence than wildtype GFP in eukaryotic cells. However, the use of EGFP as a reporter protein to quantify gene expression is limited by the sensitivity of detection. Quantification can be achieved by flow cytometry analysis of fluorescence intensity in living cells. EGFP has a single red-shifted excitation peak at $488 \mathrm{~nm}$, which is ideal for automated analysis by flow cytometry instruments using argon ion lasers and a standard FITC filter. Nevertheless, this analysis requires a large amount of cells in suspension. Alternative methods involve the preparation of cell extracts and analysis by conventional western analysis or fluorimetric techniques. In most cell types, when EGFP gene delivery is mediated by standard non-viral techniques, the efficiency of transfection is rather low, which compromises the sensitivity of EGFP quantification by either of the techniques mentioned above. The development of the laser scanning cytometer enabled the combination of single-cell detection with quantification (4). The microscope-based laser scanning cytometer permits the detection of laser-excited fluorescence emitted by individual cells on a solid support, automatic scanning of designated areas, and the generation and processing of multiparametric values.

Therefore, laser scanning cytometry offered unique characteristics to assess EGFP gene expression in morphologically distinct cultured cells. We tested its ability to quantify the gene transfer efficiency and transcriptional activity of skeletal muscle-specific promoters in both C2C12 muscle and 293 epithelial cells using EGFP as a reporter protein.

\section{MATERIALS AND METHODS}

All reagents were from Sigma (St. Louis, MO, USA) unless otherwise stated. Restriction enzymes were from Roche Molecular Biochemicals (Mannheim, Germany).

\section{Construction and Purification of Plasmids}

A derivative of plasmid pBluescript $^{\circledR}($ Stratagene, La Jolla, CA, USA) with a modified multicloning site was used to subclone the polyadenylation signal of the bovine growth hormone. The cDNA of EGFP was cut out from plasmid pEGFP-N1 (BD Biosciences Clontech, Palo Alto, CA, USA) and subcloned upstream of the polyadenylation signal, producing the promoter-less plasmid pBG (Figure 1). Next, promoter sequences from the mouse muscle creatine kinase (MCK) promoter (6) and human muscle glycogen phosphorylase (MGP) promoter (5) were subcloned upstream of EGFP (Figure 1).

Plasmids were purified using the QIAGEN ${ }^{\circledR}$ Plasmid Midi Kit (Qiagen, Hilden, Germany). Human MGP DNA was a gift from Dr. J.M. Lockyer (Tulane University). Mouse MCK was provided by J. Buskin (University of Washington).

\section{Cell Lines and Culture Conditions}

All cell lines were obtained from the ATCC (Manassas, VA, USA). C2C12 mouse myoblast cells were maintained in DMEM with $4 \mathrm{mM}$ L-glutamine supplemented with $10 \% \mathrm{FBS}, 25 \mathrm{mM}$ glucose, $100 \mathrm{U} / \mathrm{mL}$ penicillin, and 100 $\mu \mathrm{g} / \mathrm{mL}$ streptomycin under a humidified atmosphere containing $5 \% \mathrm{CO}_{2}$. Differentiation was promoted by allow ing the myoblast culture to reach confluence and replacing $10 \%$ FBS by $2 \%$ horse serum. Widespread myotube formation was achieved after three days in culture. The 293 human embryo kidney cell line was cultured in the same medium as the $\mathrm{C} 2 \mathrm{C} 12$ myoblasts.

\section{Transient Transfections and Cell Processing}

$\mathrm{C} 2 \mathrm{C} 12$ cells were grown on 8-well Permanox ${ }^{\circledR}$ slides (Nalge Nunc International, Naperville, IL, USA), allowed to reach $80 \%$ confluence, and transfected with equimolar amounts of the constructs by means of FuGENE ${ }^{\mathrm{TM}} 6$ (Roche Molecular Biochemicals) following the manufacturer's guidelines. The total amount of DNA in each transfection experiment was brought up to 


\section{Short Technical Reports}

$0.15 \mu \mathrm{g}$ using plasmid pGEM1 ${ }^{\circledR}$ (Promega, Madison, WI, USA) as a stuffer. Briefly, 0.3 $\mu \mathrm{L}$ FuGENE 6 was diluted in $10 \mu \mathrm{L}$ serum-free DMEM and left to stand at room temperature for $5 \mathrm{~min}$, supplemented with the plasmid solution (about $0.7 \mu \mathrm{L}$ ), and incubated for a further $30 \mathrm{~min}$ at the same temperature. The transfection mixture was added to each well in the slide, which contained

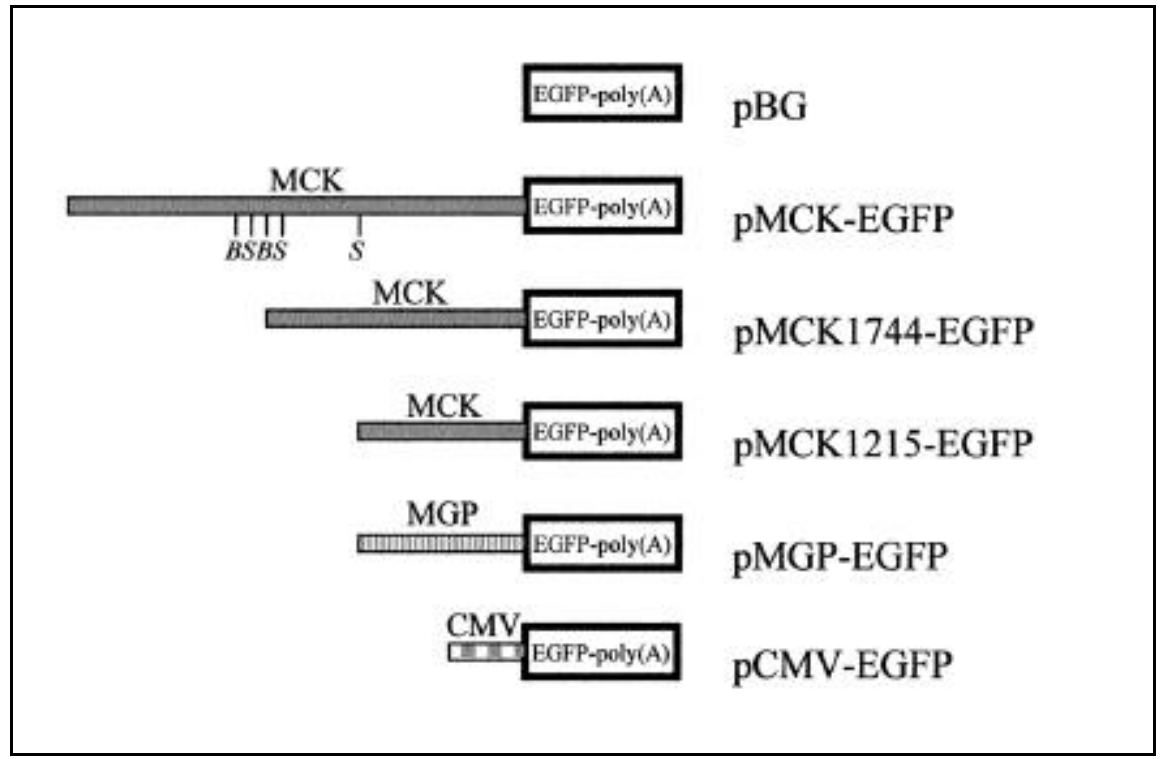

Figure 1. Schematic representation of EGFP-expressing plasmids. Nucleotide positions are relative to the transcription start site $(+1)$. The promoter-less plasmid pBG bears the EGFP cDNA upstream of the bovine growth hormone polyadenylation signal. pMCK-EGFP contains a 3-kb fragment of the mouse MCK promoter, whose $3^{\prime}$ end is nucleotide +8 . pMGP-EGFP bears a 1.2-kb fragment of the human MGP promoter, whose $3^{\prime}$ end is nucleotide +58 . pCMV-EGFP contains a $0.6-\mathrm{kb}$ fragment of the CMV promoter. Deletions of pMCK-EGFP were achieved by digestion with $M l u \mathrm{I}$ (in the polylinker) and either $B g I I$ or StuI (which cut at nucleotides -1744 and -1215 in the promoter, respectively), filled in by treatment with Klenow DNA polymerase and religated; the constructs were named pMCK1744-EGFP and pMCK1215-EGFP, respectively. Restriction mapping and sequencing were used to verify the proper cloning and orientation of DNA fragments.
$0.4 \mathrm{~mL}$ regular culture medium. Twenty-four hours after transfection, myotube formation was promoted by switching from regular medium to $2 \%$ horse serum-containing medium. Three days later, the cells were washed in PBS (supplemented with $20 \mathrm{mM}$ glycine), fixed for $30 \mathrm{~min}$ in $4 \%(\mathrm{w} / \mathrm{v})$ paraformaldehyde dissolved in PBS at room temperature, rinsed in PBS-glycine, incubated for $10 \mathrm{~min}$ in $1 \mu \mathrm{g} / \mathrm{mL}$ Hoescht 33342 to label cellular nuclei, washed twice for $5 \mathrm{~min}$ in PBS-glycine, and mounted with glass coverslips using Immuno-Fluore ${ }^{\mathrm{TM}}$ mounting medium (ICN Biomedicals, Costa Mesa, CA, USA). Transient plasmid transfection and cell fixation in the 293 line was performed essentially as described for the C2C12 line, but $40 \%$ of confluent slides were used at the time of transfection. This enabled cells to reach $100 \%$ confluence on fixation while avoiding overgrowth of the culture.

\section{Quantification of Fluorescence: Laser Scanning Cytometry Analysis}

A Laser Scanning Cytometer (LSC $\left.{ }^{\circledR}\right)$ from Compucyte (Cambridge, MA, USA) was used to detect and quantify the $509 \mathrm{~nm}$ fluorescence emitted by the cells as a result of EGFP expression. First, the slides were positioned on the computer-controlled stage
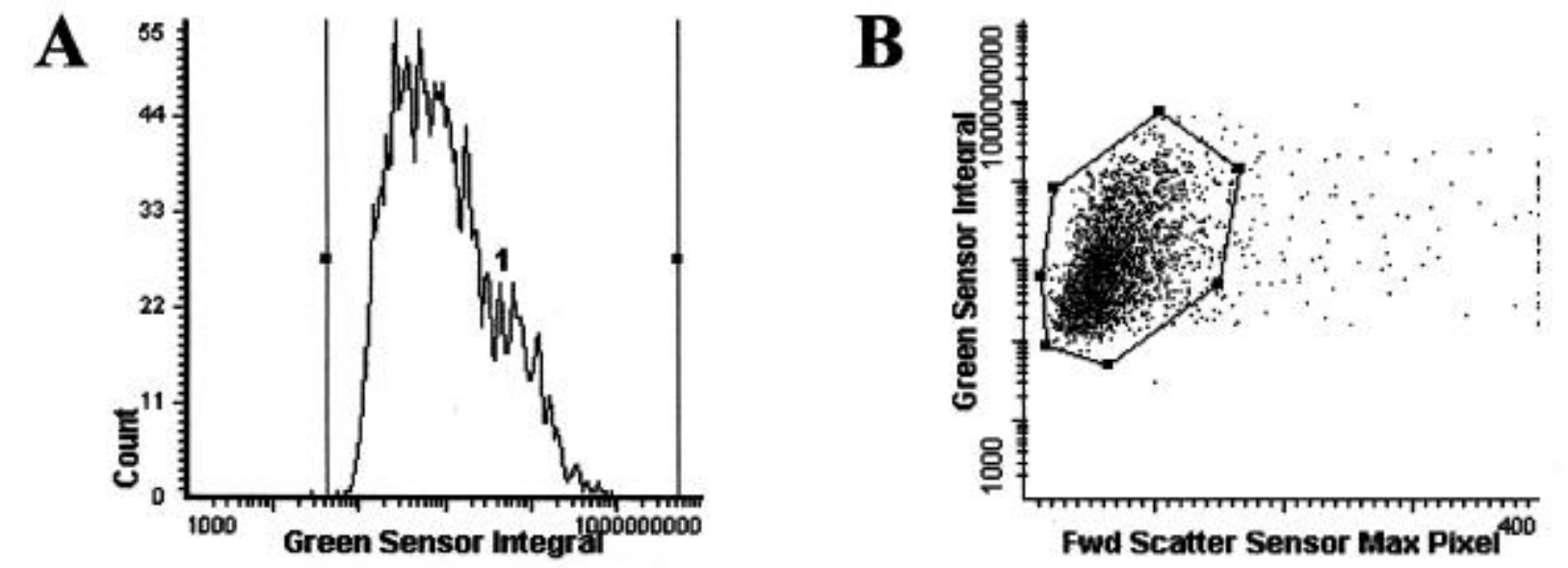

Figure 2. Identification of green fluorescent cells in a 293 cell culture transfected with pCMV-EGFP. The histogram in panel A represents the number of green fluorescent spots found in one culture and their integrated fluorescence value. This population of spots is further analyzed in panel B, in which the integrated fluorescence is plotted versus the light scattered by the particles, to discriminate between positive cells and aggregates or autofluorescent debris. The enclosed dots correspond to the EGFP-expressing cells used in subsequent measurements. 
of the LSC ${ }^{\circledR}$ microscope, and the nuclear stain Hoescht was excited by UV light to visualize the cell monolayer. Next, a $7-\mathrm{mm}^{2}$ area was scanned in every culture using a 488-nm argon ion laser with a $20 \times$ objective lens. The resulting green light emission (509 nm) above a settled threshold value was recorded and used to contour positive cells as previously described (4). The light scatter was recorded for each positive cell and used to discriminate them from aggregates and debris. Other parameters were also measured: the position of emitting area in the slide, the area within the integration contour, and the maximal pixel value of EGFP within this area. Finally, the integrated intensity of fluorescence within the contour area was used to quantify EGFP expression. Differences between groups were assessed by Student's $t$ test of the data from three independent experiments.

\section{RESULTS AND DISCUSSION}

\section{Efficiency of Gene Transfection}

To examine the efficiency of gene transfer into 293 epithelial and C2C12 muscle cells, a plasmid construct expressing EGFP under the control of the cytomegalovirus (CMV) promoter was used (Figure 1). The presence of EGFP in transfected cells was analyzed by laser scanning of defined areas $\left(7 \mathrm{~mm}^{2}\right)$ of the monolayers. The fluorescenceemitting spots were identified by setting a threshold contour value above background, the area for each positive spot was recorded, and the integrated fluorescence was calculated as the sum of intensities of all pixels within this contour (Figure 2A). Simultaneously, the light scattered by these particles was measured and plotted versus the integrated fluorescence. The resulting scattergram (Figure 2B) allowed us to discriminate and select positive single cells from aggregates and debris, which have a higher maximal scatter pixel. Random visual inspection of some of the spots displayed in Figure 2B confirmed the specificity of the selection and the adjustment of contour to cell morphology. The sum of areas of all positive cells in each monolayer was divided by the total scanned area to esti- mate the efficiency of transfection. In a confluent cell monolayer, this ratio is equivalent to that of the number of positive cells compared to total cells. As expected, the efficiency of gene transfer into the readily transfectable 293 cells was higher $(10 \% \pm 1.17 \%)$ than that of the muscle cell line $(0.65 \% \pm 0.11 \%)$.

Therefore, laser scanning cytometry has remarkable advantages for automated detection of EGFP-expressing cells over alternative systems, such as manual counting of the cells in the plate and analysis by flow cytometry. Manual counting is limited by the biased selection of positive cells, and it is timeconsuming in low efficiency transfections. Unlike laser scanning cytometry, flow cytometry requires a large number of cells and the preparation of cell suspensions. Moreover, laser scanning cytometry offers spatial resolution, thus allowing the analysis of morphologically distinct cells, such as round-shaped and muscle fibers. This feature is based on its capacity to define contour areas and to integrate fluorescence intensity.

\section{Quantification of EGFP Expression Level}

Next, we used the LSC system to assess the transcriptional activity of the skeletal muscle promoters, MCK and

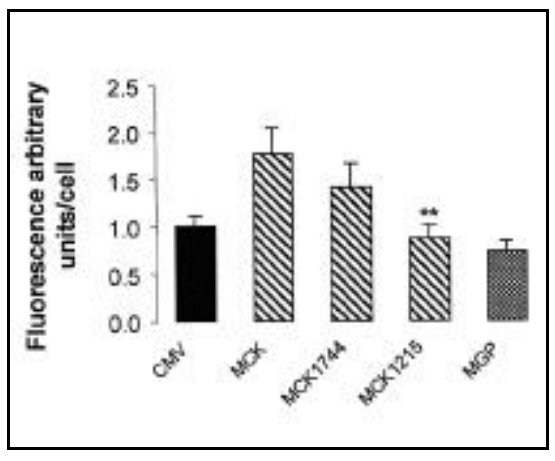

Figure 3. Integrated fluorescence value in C2C12 muscle cells transfected with EGFPexpressing constructs. $\mathrm{C} 2 \mathrm{C} 12$ myoblasts were transfected with the plasmids shown in Figure 1 and allowed to differentiate, and the fluorescence-emitting cells were identified and analyzed. The integrated fluorescence value per cell (mean \pm SEM) found in the pCMV-EGFP-transfected culture was arbitrarily assigned the value 1. The names under the bars refer to the promoter present in the construction used in each transfection. The Student's $t$ test is used in the statistical analysis $(P<0.01$ between pMCK1215-EGFPand pMCK-EGFP-transfected cells). truncated versions and MGP (Figure 1) in $\mathrm{C} 2 \mathrm{C} 12$ muscle cells. Mean values of the integrated fluorescence of positive cells were calculated for every plasmid construct and expressed relative to the value obtained with the CMV promoter. We would like to highlight that some of these data were generated by statistical analysis of 70-100 discriminated fluorescence-emitting $\mathrm{C} 2 \mathrm{C} 12$ muscle cells. Indeed, owing to the low efficiency of transfection of these cells with classical non-viral methods, the quantification analysis would not have been possible by other means using EGFP as a reporter protein. The largest 3-kb segment of the MCK promoter showed the highest transcriptional activity in C2C12 cells, which exceeded that of the CMV promoter. Deletion of MCK promoter to nucleotide -1744 slightly decreased $(20 \%)$ the expression of EGFP. In contrast, deletion to -1215 halved the EGFP levels. These results are consistent with previous studies that detected an enhancer element between nucleotides -1256 and -1020 (3). Transfection with the construct containing the MGP promoter fused to EGFP led to lower fluorescence intensity, below those of MCK and CMV.

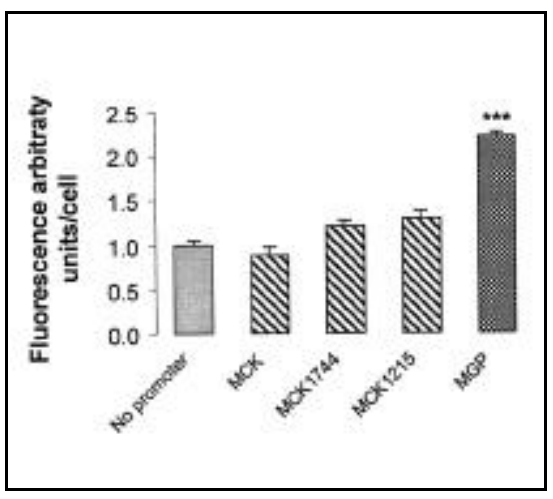

Figure 4. Integrated fluorescence value in 293 kidney cells transfected with EGFP-expressing constructs. The 293 cell monolayers were transfected with the plasmids shown in Figure 1 and allowed to differentiate, and the fluorescence-emitting cells were identified and analyzed. The names under the bars refer to the promoter present in the construction used in each transfection (the "No promoter" bar represents cells transfected with the construct $\mathrm{pBG}$ ). The integrated fluorescence value per cell (mean \pm SEM) found in the promoter-less plasmid pBG-transfected culture is arbitrarily assigned the value 1 . The Student's $t$ test was used in the statistical analysis $(P<0.001$ between pMGP-EGFP- and pBG-transfected cells). 
To study the tissue specificity of these promoters, we repeated the experiments in kidney epithelial cell line 293, using a promoter-less construct (pBG) as a control for residual activity. In this case, all MCK constructs displayed very weak transcriptional activity, to levels equal to those measured in the promoterless construct, as reported elsewhere (6). In contrast, the MGP construct displayed significant expression of EGFP, pointing to its low tissue specificity.

In summary, since laser scanning cytometry has become available, diverse applications have been described (1), such as cell-cycle analysis, enzyme kinetics, molecular binding and translocation, and FISH analysis. Here, we show that laser scanning cytometry is a highly sensitive technique for the automated assessment of gene transfer efficiency and promoter activity of EGFP constructs. Thus, laser scanning cytometry has unique features that broaden the utility EGFP as a reporter protein.

\section{REFERENCES}

1.Darzynkiewicz, Z., E. Bedner, X. Li, W. Gorczyca, and M.R. Melamed. 1999. Laserscanning cytometry: a new instrumentation with many applications. Exp. Cell Res. 249:112.

2.De Martin, R., M. Raidl, E. Hofer, and B.R. Binder. 1997. Adenovirus-mediated expression of the green fluorescent protein. Gene Ther. 4:493-495

3.Jaynes, J.B., J.E. Johnson, J.N. Buskin, C.L. Gartside, and S.D. Hauschka. 1988. The muscle creatine kinase gene is regulated by multiple upstream elements including a muscle specific enhancer. Mol. Cell Biol. 8:62-70.

4.Kamentsky, L.A. and L.D. Kamentsky. 1991. Microscope-based multiparameter laser scanning cytometer yielding data comparable to flow cytometry data. Cytometry 12:381387.

5.Lockyer, J.M. and J.B. McCracken. 1991. Identification of a tissue-specific regulatory element within the human muscle glycogen phosphorylase gene. J. Biol. Chem. 266:20262-29289.

6.Sternberg, E.A., G. Spizz, W.M. Perry, D. Vizard, T. Weil, and E.N. Olson. 1988. Identification of upstream and intragenic regulatory elements that confer cell-type-restricted and differentitation-specific expression on the muscle creatine kinase gene. Mol. Cell. Biol. 8:2896-2909.

7.Yang, T.T., L. Cheng, and S.R. Kain. 1996. Optimized codon usage and chromophore mutations provide enhanced sensitivity with the green fluorescent protein. Nucleic Acids Res. 2422:4592-5493.
This research was supported by grant no. SAF2000-0193 from the Dirección General de Investigación, Ministerio de Ciencia y Tecnología (Spain). The authors gratefully acknowledge Jaume Comas from the Servei Científico Tècnics de la Universitat de Barcelona for assistance in laser scanning cytometry analysis and Alexandra Arias for technical assistance. A.F.M. was recipient of a fellowship from Dirección General de Investigación, Ministerio de Ciencia y Tecnología (Spain). Address correspondence to Dr. Andreu Ferrer-Martínez, Departament de Bioquímica i Biologia Molecular, Universitat de Barcelona, C/ Martí i Franqués 1, E-08028, Barcelona, Catalonia, Spain. e-mail:aferrer@porthos.bio.ub.es

Received 5 June 2001; accepted 13 August 2001.

\section{Andreu Ferrer-Martínez and Anna Maria Gomez-Foix Universitat de Barcelona Barcelona, Catalonia, Spain}

For reprints of this or any other article, contact Reprints@BioTechniques.com

\section{Visualization of Whole- Mount Skeletal Expres- sion Patterns of $\mathrm{LacZ}$ Reporters Using a Tissue Clearing Protocol}

BioTechniques 32:66-73 (January 2002)

\section{ABSTRACT}

Gene targeting or trapping constructs that utilize the lacZ gene encoding $\beta$-galactosidase activity to trap promoter expression have become an increasingly important way to disrupt gene function and monitor gene expression. A number of genes targeted in this way have revealed both ex- pected and unexpected developmental abnormalities of the skeleton. The use of X-gal staining to monitor gene expression in developing skeletal structures is hampered in these mutants because, during the critical latter stages of mouse embryonic development, visualization is hindered by the opacity of overlying soft tissue. Here, we report the development of a reliable method to clear exogenous tissue in late-stage em bryos and neonates that still preserves skeletal X-gal staining patterns. This protocol reveals (i) specific cell staining in localized regions of developing bone and cartilage in two different genetic models and (ii) that the intensity of X-gal staining is consistent with the level of expression of lacZ. We conclude that this protocol accurately reflects both the specificity and intensity of expression and will facilitate the analysis of mouse skeletal development.

\section{INTRODUCTION}

The use of skeletal staining protocols allows visualization of developing skeletal structures in late-stage mouse em bryos and neonates. These stages of development are particularly important in the study of bone biology because they mark the onset of processes of endochondral and intra-membranous ossification in the embryo. The standard protocol used to study these processes involves the use of Alizarin red and Alcian blue dyes. These preferentially stain bone and cartilage, respectively, which, when followed by treatment with potassium hydroxide to clear exogenous tissue, present a colorful view of the entire developing skeleton against a background of transparent soft tissue $(5,6)$. This technique has been used to monitor not only the progression of skeletal development in normal mice but also the consequences of genetic mutations on these structures $(1,7-9,11-13)$.

Genetic modification of mice by gene targeting and trapping techniques has had a major impact on biological research in revealing the consequences of gene loss. Adaptations of these techniques to include the use of marker genes to report on promoter expression patterns of targeted genes have been particularly useful to the study of spatial and temporal developmental regulation of gene expression. The genetic marker 